\title{
Effectiveness of financial incentives to improve adherence to maintenance treatment with antipsychotics: cluster randomised controlled trial
}

\begin{abstract}
Stefan Priebe professor of social and community psychiatry ${ }^{1}$, Ksenija Yeeles research fellow ${ }^{2}$, Stephen Bremner lecturer in medical statistics ${ }^{3}$, Christoph Lauber medical director ${ }^{4}$, Sandra Eldridge professor of biostatistics ${ }^{3}$, Deborah Ashby professor of medical statistics and clinical trials ${ }^{5}$, Anthony S David professor of cognitive neuropsychiatry ${ }^{6}$, Nicola O'Connell research assistant ${ }^{1}$, Alexandra Forrest research assistant ${ }^{2}$, Tom Burns professor of social psychiatry ${ }^{2}$

'Unit for Social and Community Psychiatry, Queen Mary University of London, Newham Centre for Mental Health, London E13 8SP, UK; ${ }^{2}$ Department of Psychiatry, University of Oxford, Warneford Hospital, Oxford, UK ; ${ }^{3}$ Centre for Primary Care and Public Health, Blizard Institute, Barts and The London School of Medicine and Dentistry, London, UK ; ${ }^{4}$ Services psychiatriques, Jura bernois, Bienne-Seeland, Bellelay, Switzerland ; ${ }^{5}$ School of Public Health, Imperial College London, St Mary's Campus, London, UK; ${ }^{6}$ Department of Psychosis Studies, Institute of Psychiatry, King's College London, UK
\end{abstract}

\begin{abstract}
Objective To test whether offering financial incentives to patients with psychotic disorders is effective in improving adherence to maintenance treatment with antipsychotics.

Design Cluster randomised controlled trial.

Setting Community mental health teams in secondary psychiatric care in the United Kingdom.

Participants Patients with a diagnosis of schizophrenia, schizoaffective disorder, or bipolar disorder, who were prescribed long acting antipsychotic (depot) injections but had received $75 \%$ or less of the prescribed injections. We randomly allocated 73 teams with a total of 141 patients. Primary outcome data were available for 35 intervention teams with 75 patients ( $96 \%$ of randomised) and for 31 control teams with 56 patients $(89 \%$ of randomised).
\end{abstract}

Interventions Participants in the intervention group were offered $£ 15$ (€17; \$22) for each depot injection over a 12 month period. Participants in the control condition received treatment as usual.

Main outcome measure The primary outcome was the percentage of prescribed depot injections given during the 12 month intervention period.

Results 73 teams with 141 consenting patients were randomised, and outcomes were assessed for 131 patients (93\%). $\Downarrow$ Average baseline adherence was $69 \%$ in the intervention group and $67 \%$ in the control group. During the 12 month trial period adherence was $85 \%$ in the intervention group and $71 \%$ in the control group. The adjusted effect estimate was $11.5 \%$ (95\% confidence interval $3.9 \%$ to $19.0 \%, \mathrm{P}=0.003$ ). A secondary outcome was an adherence of $\geq 95 \%$, which was achieved in $28 \%$ of the intervention group and $5 \%$ of the control group (adjusted odds ratio $8.21,95 \%$ confidence interval 2.00 to $33.67, \mathrm{P}=0.003$ ). Although differences in clinician rated clinical improvement between the groups failed to reach statistical significance, patients in the intervention group had more favourable subjective quality of life ratings $(\beta=0.71,95 \%$ confidence interval 0.26 to $1.15, P=0.002$ ). The number of admissions to hospital and adverse events were low in both groups and did not show substantial differences.

Conclusion Offering modest financial incentives to patients with psychotic disorders is an effective method for improving adherence to maintenance treatment with antipsychotics.

Trial registration Current Controlled Trials ISRCTN77769281.

\section{Introduction}

Poor adherence to antipsychotic drugs is a major problem in patients with psychotic disorders, linked to increased rates of readmission to hospital and high treatment costs. ${ }^{1}$ Between $25 \%$ and $80 \%$ of patients fail to take their drugs correctly at some point in their treatment. ${ }^{2}$ Poor adherence to treatment can treble the costs of external services. ${ }^{3}$ Many interventions have been tried to improve adherence to treatment in patients with psychotic disorders, including compliance therapy, psychotherapy, family education, telephone prompting, and psychoeducation. However, reviews of such interventions find at best only modest effects, and evidence that any intervention significantly improves drug adherence in non-adherent patients with psychotic disorders in the community is inconsistent. ${ }^{45}$ 
Indeed, adherence therapy, a client centred approach that aims to improve adherence by applying cognitive behavioural methods, is specifically not recommended for people with schizophrenia and has no clear benefits for patients. ${ }^{67}$ Psychoeducation has been suggested to improve adherence in patients with bipolar disorders but not in those with non-affective psychoses. ${ }^{8}$ Against this background, financial incentives have been considered in such patients to improve adherence to drugs.

Systematic reviews of the effectiveness of financial incentives suggest that they can improve adherence to a range of medical treatments. ${ }^{9}{ }^{10}$ In treatments of mental health, financial incentives have been shown to encourage attendance at treatment sessions and outpatient clinics and abstinence from smoking or substance misuse. ${ }^{11-25}$ Observational studies with small samples suggest that financial incentives may also improve drug adherence in patients with psychotic disorders, yet to date this has not been tested in a trial. ${ }^{23}{ }^{26}$ The idea of using financial incentives to increase adherence to antipsychotic drugs ${ }^{27}$ is controversial and has been debated, ${ }^{28-31}$ and in surveys clinicians have raised concerns about it. ${ }^{32}{ }^{33}$ In a focus group study, different stakeholder groups in the United Kingdom identified clinical effectiveness as critical and highlighted the need for this to be established by systematic research. ${ }^{34}$

We conducted a randomised controlled trial to test whether offering modest financial incentives can improve adherence to maintenance treatment with antipsychotics in patients with psychotic disorders and poor adherence.

\section{Methods}

The study was a cluster randomised controlled trial with a 1:1 allocation ratio of mental health teams to intervention or control arm. We tested the hypothesis that offering financial incentives to patients with psychotic disorders with suboptimal adherence would improve adherence to maintenance treatment with antipsychotics.

To prevent contamination of practice within teams and to facilitate the assessment of overall experiences in teams with the practice we allocated teams, and not individual patients, at randomisation. This also made it possible for teams in the intervention group to offer financial incentives to other patients outside the study, without compromising the study design.

Teams in the intervention group were therefore in a position to avoid a potential sense of unfairness among patients cared for by the same team and being excluded from receiving incentives through the study design.

Eligible mental health teams were community mental health teams and assertive outreach teams in England and Wales. Mental health care in the United Kingdom has been strictly geographically sectorised for several decades, with multidisciplinary teams responsible for all patients from defined geographical areas. The basic model is for community mental health teams to take all referrals from general practitioners serving a population of between 20000 and 70000 . Such teams carry a mixed caseload of between 200 and 300 patients, of whom over half will have long term psychotic disorders. Assertive outreach teams carry caseloads of 60-100 patients who are mostly psychotic and difficult to engage, referred to them from community mental health teams. Of these a significant proportion (often $20-40 \%$ in community mental health teams and over $50 \%$ in assertive outreach teams) will receive depot treatment.

To be included patients had to be under the care of the team for at least four months; aged between 18 and 65 years; have a diagnosis of schizophrenia, schizoaffective disorder, or bipolar disorder according to the international classification of diseases (10th revision) ${ }^{35}$ be prescribed for long acting (depot) injections of antipsychotic drugs; show poor adherence to antipsychotic drugs - that is, received $75 \%$ or less of prescribed depot injections over the four months before screening; and have the capacity to provide informed consent to participate in the study. Exclusion criteria were intellectual disability and poor command of English.

Originally we had defined an adherence level of $50 \%$ or less as an inclusion criterion. When discussing the study with clinical teams, clinicians stated that services would usually prevent adherence levels dropping below $50 \%$ in patients with clinical risks who were receiving long acting depot injections, and emphasised that adherence levels between $50 \%$ and $75 \%$ usually indicate a significant failure in achieving treatment adherence, often prompting immediate interventions. In line with this, we found low numbers of patients with adherence levels of 50\% or less during screening. With the support of the trial steering committee and the agreement of the funder we subsequently changed the inclusion criterion to an adherence level of $75 \%$ or less. For the most frequent cycle of fortnightly prescriptions, this reflects missing one injection or more every two months.

\section{Intervention}

Patients in the intervention group were offered $£ 15$ for each injection of antipsychotic drug over a 12 month period (intervals between injections ranging from one to four weeks). Clinicians gave the monetary incentive in cash directly to patients after each injection, and patients signed a receipt. We chose the standard sum of $£ 15$ because a fixed sum per injection simplifies the practice, it is below the limit of $£ 20$ per week over which there would be interference with patients' entitlements to disability benefits, and it was considered an important incentive without inducing financial dependency.

Patients in teams allocated to the control condition received treatment as usual with no financial or other incentive for taking their drugs. The type, frequency, and dosage of drugs and all other interventions were not affected by participation in the study.

\section{Outcomes}

The primary outcome was the percentage of prescribed depots received in the community within the 12 month trial period. The percentage was based on the total number of prescribed and actually received depots in the defined period, independently of potential delays in administering the depot. Assuming that adherence during admission to hospital and imprisonment was affected by context factors, we excluded such periods unless they were shorter than one depot cycle.

Secondary outcomes were the percentage of patients with at least $95 \%$ adherence, which was meant to reflect practically full adherence (allowing for one depot per year to be missed in patients receiving fortnightly prescriptions); patients' clinical global improvement at the end of the 12 month study period, dichotomised as "improved" versus "no change, or worse" from the clinical improvement component of the clinical global impression scale ${ }^{36}$ ranging from 1 (very much improved) to 7 (very much worse), rated by the consultant psychiatrist or other clinician; patients' subjective quality of life rated on the DIALOG scale,$^{37}$ which has eight items on satisfaction with different life domains, ranging from 1 (lowest satisfaction) to 7 (highest satisfaction) and has been shown to have good psychometric properties ${ }^{38}$ in patients living in the community 
with psychotic disorders; patients' satisfaction with drug rated on one item, also contained in the DIALOG scale, and ranging from 1 to 7 ; number of involuntary and voluntary hospital admissions and adverse events during the 12 month period (that is, attempted and completed suicides, incidents of physical violence, and police arrests); and time spent in work, training, and education.

We recorded all adverse events, including those possibly attributable to the intervention. All data other than clinical global impression and DIALOG were obtained from patient medical records. We collected data going back 12 months from the date of randomisation (baseline), and then again going back 12 months from 372 days after randomisation, allowing seven days after randomisation for the intervention to begin. The only exception to this was the baseline depot card data, which we collected going back 12 months from the date of screening.

The protocol had prespecified further secondary outcomes that are not reported here. These concern cost effectiveness, since this would require the reporting of much additional and complex information about the utilisation of formal and informal support; slippage - that is, delays of received injections compared with the prescribed dates, as such delays are a more meaningful outcome, if adherence levels do not differ (that is, if the two groups did not significantly differ in the primary outcome, which is reported in this paper); and 18 months' outcomes, because outcomes beyond the 12 month study period were influenced by additional factors that were no longer experimentally controlled. After the 12 month period clinicians in the intervention teams were free to continue or discontinue offering incentives, and clinicians in the control teams could decide to offer incentives to their patients.

\section{Recruitment procedure}

We approached both community mental health teams and assertive outreach teams in England and Wales. Researchers then visited interested teams to discuss the study. Written informed consent to participate was obtained from team managers or consultant psychiatrists, or both.

A team clinician approached eligible patients in participating teams and, if they agreed, they were contacted by a researcher who explained the study in detail, obtained written informed consent, and asked them to rate their subjective quality of life. After this one-off contact, the participants were not required to provide any more information or be contacted by a researcher again. We expected that patients with psychosis and poor adherence to the prescribed treatment would be difficult to recruit to research, in particular to randomised controlled trials like this one in which they could be allocated to a control condition with no personal benefit. We therefore minimised the burden on patients and did not require them to participate in any research interview after providing written informed consent. Any more requirements could have made the study more difficult to implement and introduced an additional selection bias. As a result, we obtained basic clinical characteristics such as diagnosis and length of illness from clinicians and records but did not assess the participants for symptoms. Only if patients volunteered to be contacted again at the end of the trial would a researcher attempt such contact to ask them to rate their subjective quality of life.

After all eligible participants in one team had been contacted and consent obtained, the team was randomised and a researcher informed them of their allocation.

\section{Sample size}

We initially assumed that three participants in each team would provide data, that the cluster size would be almost constant, and that the intracluster correlation coefficient would be 0.05 . To detect a shift in mean adherence from $65 \%$ to $85 \%$ (assuming a standard deviation in each group of $30 \%$ ) with $90 \%$ power at the $5 \%$ significance level, we estimated that we would require 47 participants in each group. We inflated this to 68 participants in each group (four patients in each of 17 clusters) to allow for clustering and drop out. Thus we planned to recruit 136 participants in total from about 68 teams. During the trial it became clear that the average cluster size was smaller, with a mean of only two patients for each cluster, but cluster sizes were more variable. In addition, the standard deviation of the baseline adherence of patients was smaller than that assumed in the original sample size calculation. In the light of these changes we reviewed the sample size calculation. However, when all the changes in the inputs to the calculation were considered, the sample size required was virtually unchanged and so no changes were made.

\section{Randomisation and masking}

An independent statistician at the Pragmatic Clinical Trials Unit, Queen Mary University of London, randomised the teams. The teams were stratified according to the deprivation of the area in which they operated, based on national data from the mental illness needs index score (low $v$ high) ${ }^{39}$ as teams in areas with higher deprivation were supposed to have more eligible participants, and possibly more challenging ones. A sequence of allocations in each stratum was generated using the "ralloc" command in Stata. ${ }^{40}$ Sixty group allocations were generated for each stratum, arranged in blocks of random length $(2,4$, or $6)$, with a randomisation ratio of 1:1.

It was not possible to blind participants or clinicians, because receipt of an incentive cannot be disguised. The primary outcome and also secondary outcomes, with the exceptions of the clinician rated global clinical improvement and the participants' rated subjective quality of life, were obtained from the medical records and should not be biased by lack of clinician masking.

\section{Statistical analysis}

A substantial delay usually occurred between the screening of participants and randomisation of the teams. We calculated the periods in hospital and in prison and baseline adherence for the baseline period. In calculating adherence, we considered treatment cycles, changes of cycles, and periods without depot prescription.

We modelled the effect on the primary outcome (percentage adherence) using a linear mixed effects regression model with a random effect for clinical treatment team. In the main analysis we used data from all participants who had at least four months of complete data during both the baseline and the intervention periods, on an intention to treat basis. We also carried out an analysis excluding patients with protocol violations for diagnoses not meeting the inclusion criteria, or more than $75 \%$ adherence in the four months before screening, using the same model specification as described. Further sensitivity analyses were conducted without adjustment for baseline adherence, excluding all patients with more than $75 \%$ adherence in the whole baseline period (rather than only four months), and only for participants with a diagnosis of schizophrenia.

We adjusted all models assessing the effect of the intervention — on both primary and secondary outcomes - for 
category of mental illness needs index score and average baseline prescription cycle. Except for clinical global improvement, which was measured only at the end of the trial, we also included the baseline measure of the outcome. In the absence of any hypothesis about a moderating effect of a specific sociodemographic variable, we did not plan to adjust for sociodemographic variables.

The effects on the binary secondary outcomes of achieving at least $95 \%$ adherence and improvement on the clinical global improvement scale were modelled using mixed effects logistic regression. We estimated the effect on subjective quality of life using a random effects model fitted by generalised least squares, since the mixed model did not converge. Admissions to hospital and adverse events were expected to be infrequent, and we therefore report the differences descriptively.

\section{Results}

Of 540 teams approached, 184 consented to take part in the study (figure $\Downarrow$ ). Overall, 73 teams with 141 consenting patients were randomised between 16 April 2010 and 15 November 2011. Ninety three consenting teams had no eligible patients, and in a further 18 teams we failed to recruit eligible patients.

Two patients withdrew directly after randomisation to the control group. One patient recruited to the control group was subsequently found to be ineligible during the baseline data collection because antipsychotic depot treatment had not been prescribed at the time of recruitment. Four patients did not meet the inclusion criteria for diagnosis but were included in the analysis. The original screening information on adherence in the previous four months was taken from a range of sources. This information was later checked against the records, and seven participants in the intervention group and four in the control group were found to be adherent. These participants were also included in the primary analysis but, together with the four with excluded diagnoses, were removed for the per protocol analysis. Table $1 \Downarrow$ lists the baseline characteristics of the sample.

The characteristics of the two groups, including their length of illness, were similar. Thirty five intervention group teams with 75 patients and 31 control group teams with 56 patients provided primary outcome data. These data were missing on average for $7 \%$ of the time during the one year study period in the intervention group and for $14 \%$ of the time in the control group. Of those, 32 teams with 71 patients from the intervention group and 30 teams with 52 patients from the control group had sufficient data on adherence during both baseline and intervention period and were included in the primary analysis (figure).

Out of the 131 patients with primary outcome data, three (2\%) were prescribed an injection every week (two in the intervention group, 3\%; one in the control group, $2 \%$ ) during the one year study period. Eighty $(61 \%)$ were prescribed an injection every two weeks $(n=51,68 \% ; n=29,52 \%)$, seven $(5 \%)$ every three weeks $(\mathrm{n}=4,5 \% ; \mathrm{n}=3,5 \%)$, and $31(24 \%)$ every four weeks $(n=13,17 \% ; n=18,32 \%)$. For $10(8 \%)$ patients the prescription cycle varied $(n=5,7 \% ; n=5,9 \%)$. The average prescription cycle for the study period was similar to that of the baseline period (table 1).

\section{Primary outcome}

Table $2 \Downarrow$ shows the effect of financial incentives on adherence to treatment. Using data from all 131 patients with endpoint data, the unadjusted difference in adherence was $14 \%$.
Adherence to treatment was significantly higher in the intervention group than in the control group (adjusted difference in means $(\beta) 11.5 \%, 95 \%$ confidence interval $3.9 \%$ to $19.0 \%$, $\mathrm{P}=0.003)$, representing on average and after adjustment for covariates $11.5 \%$ greater adherence in the group receiving financial incentives.

\section{Sensitivity analyses}

The results were similar between the analysis excluding those with incorrect diagnoses or more than $75 \%$ adherence in the four months before screening ( $\beta=10.7 \%, 95 \%$ confidence interval $3.0 \%$ to $18.5 \%, \mathrm{P}=0.006$ ) and the sensitivity analysis without adjustment for baseline adherence ( $\beta=11.6 \%, 3.7 \%$ to $19.5 \%, \mathrm{P}=0.004)$. Excluding all patients with more than $75 \%$ adherence for the whole baseline period gave an effect estimate of $15.7 \%$, and restricting the analysis only to patients with a diagnosis of schizophrenia gave an estimate of $10.7 \%$.

\section{Secondary outcomes}

Table 2 also shows the effect of financial incentives on the secondary outcomes. For all 131 patients with primary outcome data, the unadjusted odds ratio of achieving at least $95 \%$ adherence was 7.11. The percentage of patients achieving at least $95 \%$ adherence during the intervention period was significantly higher in the intervention group than in the control group (adjusted odds ratio 8.21, 95\% confidence interval 2.00 to $33.67, \mathrm{P}=0.003$ ), as was participants' subjective rating of quality of life ( $\beta=0.71,95 \%$ confidence interval 0.26 to 1.15 , $\mathrm{P}=0.002)$. Differences on clinician rated clinical improvement were not statistically significant, although the odds of being rated as improved were 2.73 times greater in the intervention condition (95\% confidence interval 0.64 to $11.59, \mathrm{P}=0.174$ ). The number of admissions to hospital and adverse events were low in both groups and did not show substantial differences.

No patient committed suicide in either group. Five patients in the intervention group (one in the control group) were in employment during the study period (intervention group: four consistently, one for 18 days; control group: one for 26 days). No patient was in formal education, and seven patients in the intervention group (five in control group) attended some type of training course.

\section{Discussion}

Offering financial incentives to patients with psychotic disorders who have poor adherence to maintenance treatment with antipsychotics is effective in improving adherence. In this study, the average baseline adherence of $67 \%$ improved by only $4 \%$ in the control group. Thus it remained largely unchanged, which might be expected in people with severe psychotic disorders and poor adherence. In the intervention group, offering financial incentives led to an average adherence of $85 \%$. Given the ceiling effect of $100 \%$ adherence, this shift represents more than half of the potentially possible maximum improvement. In the intervention group, $21(28 \%)$ achieved adherence of at least 95\% compared with three (5\%) in the control group.

\section{Strengths and weaknesses of this study}

The trial was conducted in a large number of teams. Outcome data were available for $131(93 \%)$ randomised participants and statistically analysed for $123(87 \%)$. Sensitivity analyses showed that the findings on the primary outcome in the intervention group were robust to a variety of assumptions. 
The study has some weaknesses. Several protocol violations occurred and data were missing on depot treatment during the baseline and study period. Blinding of clinicians, patients, and researchers was not possible, and less than $50 \%$ of the final sample had patient reported outcomes. The sample was confined to those patients receiving long acting depot injections so the results may not be generalisable to those receiving other formulations, particularly as the practice of offering financial incentives for patients taking oral drugs would be more complicated. The study had no active control, and potential expectancy effects of the clinicians may have influenced practice and outcomes. All participating teams were willing to offer financial incentives, at least within an experimental study, and it remains unclear whether financial incentives would be similarly effective when provided by those teams that did not consent to participate in the study, usually because clinicians or managers expressed an unwillingness to try financial incentives.

\section{Comparison with previous studies}

Previous research suggested that financial incentives might be effective in influencing the health behaviour of patients with severe mental illness, and small observational studies reported that this might also apply to adherence to maintenance treatment with antipsychotics. We report the first randomised controlled trial testing this hypothesis, providing evidence that offering financial incentives is effective in improving treatment adherence in this patient group.

\section{Implications and explanations of findings}

Did the improved adherence result in better clinical outcomes in this study? The difference on clinician rated improvement was not significant. Admissions to hospital and adverse events were rare in both groups hence limiting the power of the study to detect differences for these variables. Yet patients in the intervention group reported a significantly more favourable subjective quality of life. Consistent treatment may have helped patients to organise their lives better, cope with problems, and engage in satisfying activities. Other factors may also have contributed to the improved subjective quality of life. Patients in the intervention group had between $£ 15$ and $£ 60$ (depending on the depot cycle) more to spend every month. This could have made a difference to patients who received social benefits. It can also be speculated that receiving money from services had a psychological effect in some patients, increased their confidence, and facilitated a more positive view of life.

Although those patients offered financial incentives were more likely to have $95 \%$ or more adherence, practically complete adherence was achieved in only $28 \%$ of these patients. The incentive of $£ 15$ per injection used in this study did not provide a solution for all patients with problematic adherence to maintenance treatment with antipsychotics. However, we can conclude that offering a modest financial incentive is the most effective method shown so far of improving treatment adherence in this patient group. It should be considered if poor treatment adherence poses a risk to patients' health and social outcomes and if other methods to achieve adherence have failed.

Community mental health teams and assertive outreach teams in the National Health Service aim to follow up patients with clinical risks and problematic adherence in the community, and to do this proactively and consistently over longer periods of time. In this context, we recruited on average about one patient in each team who consented to participate. The low number suggests that the practice of community mental health teams and assertive outreach teams may be effective in ensuring good adherence to long acting injections with most patients. How many patients will qualify to be offered financial incentives if the practice is rolled out? This is difficult to predict with precision as eligibility in routine practice can be more flexibly assessed than in rigorous research trials. If the clinical eligibility criteria in this study are identically applied, however, teams are likely to have on average no more than one or two eligible patients at any time. This means that financial incentives may be offered to fewer than about 1800 patients in the NHS across England. This is a substantial, but limited, number, and whether more patients may qualify in service contexts other than the one provided by the NHS can only be speculated.

The ethical issues of offering financial incentives to increase adherence to antipsychotic treatment have been discussed elsewhere. The evidence for the effectiveness of incentives provided in this study may inform the debate. All participants in this study had consented to take their treatment and had a certain level of adherence at baseline. Thus, financial incentives helped them to overcome organisational and other problems that had previously prevented them from more regular adherence. However, incentives in our study were not used to persuade otherwise non-consenting patients to accept treatment.

\section{Future research}

Whether offering higher financial incentives would be ethically acceptable and more effective, remains unclear. Further research should test the longer term impact of offering financial incentives. Qualitative research is required to identify the psychological mechanisms that may explain the positive effect of offering financial incentives on adherence and explore whether the effect on subjective quality of life may result from wider benefits for the engagement of patients and not only improved adherence.

Members of the full Financial Incentives for Adherence Trial (FIAT) research group:Richard Ashcroft, Claudia Ashton, Deborah Ashby, Kerri Bailey, Kirsten Barnicot, Stephen Bremner, Tom Burns, Anthony David, Philip Eldridge, Sandra Eldridge, Mike Firn, Alexandra Forrest, Connie Geyer, Elizabeth Highton-Williamson, Catherine Henderson, Lauren Kelley, Martin Knapp, Christoph Lauber, Helen Morley, Nicola O'Connell, Stefan Priebe, Sarah Watkins, and Ksenija Yeeles.

The study protocol can be accessed at www.biomedcentral.com/1471244X/9/61.

Contributors: SP (chief investigator) conceived and designed the study, developed the intervention, managed the study, and monitored recruitment and data collection for the London site and the whole trial. $\mathrm{He}$ is the guarantor. KY managed the study, developed the intervention, designed the data collection tools, monitored recruitment for the Oxford site and data collection for the whole trial, and managed and cleaned the data. SB designed and conducted the statistical analysis and supervised the study. CL conceived, designed, and managed the study, developed the intervention, and monitored recruitment for the Liverpool site. SE and DA conceived, designed, and supervised the study, and designed the statistical analysis. ASD conceived, designed, and supervised the study. NO'C and AF implemented the study and managed the recruitment, data collection, and data. TB conceived, designed, and managed the study, developed the intervention, and monitored recruitment and data collection for the Oxford site. All authors helped write and review the report.

Funding: This trial was funded by the National Institute for Health Research Health Technology Assessment (NIHR HTA) programme (project No 07/60/43) and will be published in full in Health Technology Assessment. The views and opinions expressed therein are those of 


\section{What is already known on this topic}

Interventions to improve adherence to maintenance treatment with antipsychotics in patients with psychosis have been relatively ineffective

The use of financial incentives has been suggested and supported by case reports

No randomised trial has been done to test whether such incentives are effective for improving medication adherence in patients with psychosis

\section{What this study adds}

Offering modest financial incentives was an effective method for improving adherence to long acting antipsychotic (depot) injections in patients with psychosis

Adherence to treatment was significantly higher for those receiving financial incentives than those in the control group

Using data from all 131 patients with endpoint data, the unadjusted difference in adherence was $14 \%$

the authors and do not necessarily reflect those of the HTA programme, NIHR, NHS, or the Department of Health. The trial is registered with ISRCTN (SRCTN77769281) and is adopted into the UKCRN and MHRN portfolios. The funder had no role in study design, data collection, data analysis, interpretation, writing of the report, or decision to submit for publication. All study researchers were independent from a funder. All authors had full access to all of the data (including statistical reports and tables) in the study and can take responsibility for the integrity of the data and the accuracy of the data analysis.

Competing interests: All authors have completed the ICMJE uniform disclosure form at www.icmje.org/coi_disclosure.pdf and declare: no support from any organisation for the submitted work; ASD received personal fees from Janssen-Cilag and Eli Lilly and TB received lecture fees from Janssen and Otsuka during the conduct of the study; no other relationships or activities that could appear to have influenced the submitted work.

Ethical approval: This study was approved by the NRES Ealing and West London Research Ethics Committee (reference No 09/H0710/35). Data sharing: Patient level data and the statistical code are available from the corresponding author at s.priebe@qmul.ac.uk. Consent for data sharing was not obtained but the presented data are anonymised and the risk of identification is low.

Declaration of transparency: The lead author affirms that this manuscript is an honest, accurate, and transparent account of the study being reported; that no important aspects of the study have been omitted; and that any discrepancies from the study as planned (and, if relevant, registered) have been explained. The lead author in this statement is the study guarantor.

1 Novick D, Haro JM, Suarez D, Perez V, Dittmann RW, Haddad PM. Predictors and clinical consequences of non-adherence with antipsychotic medication in the outpatient treatment of schizophrenia. Psychiatry Res 2010;176:109-13.

2 Conley RR, Kelly DL. Management of treatment resistance in schizophrenia. Biol Psychiatry 2001;50:898-911

3 Knapp M, King D, Pugner K, Lapuerta P. Non-adherence to antipsychotic medication regimens: associations with resource use and costs. Br J Psychiatry 2004:184:509-16.

4 McDonald HP, Garg AX, Haynes RB. Interventions to enhance patient adherence to medication prescriptions-scientific review. JAMA 2002;288:2868-79.

5 Nose M, Barbui C, Gray R, Tansella M. Clinical interventions for treatment non-adherence in psychosis: meta-analysis. Br J Psychiatry 2003;183:197-206.

6 Gray R, Leese M, Bindman J, Becker T, Burti L, David A, et al. Adherence therapy for people with schizophrenia. European multicentre randomised controlled trial. BrJ Psychiatry 2006:189:508-14.

7 National Collaborating Centre for Mental Health. Schizophrenia: core interventions in the treatment and management of schizophrenia in adults in primary and secondary care (Update). CG82. March 2009 ed. National Institute for Health and Clinical Excellence, 2009:41.

8 Vieta E. Improving treatment adherence in bipolar disorder through psychoeducation. $J$ Clin Psychiatry 2005;66(suppl 1):24-9.

9 Giuffrida A, Torgerson DJ. Should we pay the patient? Review of financial incentives to enhance patient compliance. BMJ 1997;315:703-7.

10 Marteau TM, Ashcroft RE, Oliver A. Using financial incentives to achieve healthy behaviour. BMJ 2009;338:b1415.

11 Burton A, Marougka S, Priebe S. Do financial incentives increase treatment adherence in people with severe mental illness? A systematic review. Epidemiol Psichiatr Soc 2010;19:233-42.

12 Carey KB, Carey MP. Enhancing the treatment attendance of mentally ill chemical abusers. $J$ Behav Ther Exp Psychiatry 1990;21:205-9.

13 Helmus TC, Saules KK, Schoener EP, Roll JM. Reinforcement of counseling attendance and alcohol abstinence in a community-based dual-diagnosis treatment program: a feasibility study. Psychol Addict Behav 2003:17:249-51.
14 Post EP, Cruz M, Harman J. Incentive payments for attendance at appointments for depression among low-income African Americans. Psychiatr Serv 2006:57:414-6.

15 Roll JM, Higgins ST, Steingard S, McGinley M. Use of monetary reinforcement to reduce the cigarette smoking of persons with schizophrenia: a feasibility study. Exp Clin Psychopharmacol 1998;6:157-61

16 Roll JM, Chermack ST, Chudzynski JE. Investigating the use of contingency management in the treatment of cocaine abuse among individuals with schizophrenia: a feasibility study. Psychiatry Res 2004;125:61-4.

17 Shaner A, Roberts LJ, Eckman TA, Tucker DE, Tsuang JW, Wilkins JN, et al. Monetary reinforcement of abstinence from cocaine among mentally ill patients with cocaine dependence. Psychiatr Serv 1997;48:807-10.

18 Sigmon SC, Steingard S, Badger GJ, Anthony SL, Higgins ST. Contingent reinforcement of marijuana abstinence among individuals with serious mental illness: a feasibility study. Exp Clin Psychopharmacol 2000:8:509-17.

19 Tidey JW, O'Neill SC, Higgins ST. Contingent monetary reinforcement of smoking reductions, with and without transdermal nicotine, in outpatients with schizophrenia. Exp Clin Psychopharmacol 2002;10:241-7.

20 Tracy K, Babuscio T, Nich C, Kiluk B, Carroll KM, Petry NM, et al. Contingency management to reduce substance use in individuals who are homeless with co-occurring psychiatric disorders. Am J Drug Alcohol Abuse 2007;33:253-8.

21 Olson RP, Greenber DJ. Effects of contingency-contracting and decision-making groups with chronic mental patients. J Consult Clin Psychol 1972;38:376-83.

22 Drebing CE, Van Ormer EA, Krebs C, Rosenheck R, Rounsaville B, Herz L, et al. The impact of enhanced incentives on vocational rehabilitation outcomes for dually diagnosed veterans. J Appl Behav Anal 2005;38:359-72.

23 Claassen D, Fakhoury WK, Ford R, Priebe S. Money for medication: financial incentives to improve medication adherence in assertive outreach. Psychol Bull 2007;31:4-7.

24 Tidey JW. Using incentives to reduce substance use and other health risk behaviors among people with serious mental illness. Prev Med 2012;55:S54-60.

25 Ries RK, Dyck DG, Short R, Srebnik D, Fisher A, Comtois KA. Outcomes of managing disability benefits among patients with substance dependence and severe mental illness. Psychiatr Serv 2004:55:445-7.

26 Staring $A B$, Mulder $C L$, Priebe S. Financial incentives to improve adherence to medication in five patients with schizophrenia in the Netherlands. Psychopharmacol Bull 2010;43:5-10.

27 Wise J. NICE Citizens Council debates incentives for healthy behaviour. BMJ 2010;340:c2747.

28 Burns T. Is it acceptable for people to be paid to adhere to medication? Yes. BMJ 2007;335:232.

29 Shaw J. Is it acceptable for people to be paid to adhere to medication? No. BM 2007;335:233

30 Cookson R. Should disadvantaged people be paid to take care of their health? Yes. BMJ 2008;337:a589.

31 Popay J. Should disadvantaged people be paid to take care of their health? No. BMJ 2008;337:a594.

32 Claassen D. Financial incentives for antipsychotic depot medication: ethical issues. JMed Ethics 2007;33:189-93.

33 Promberger M, Brown RC, Ashcroft RE, Marteau TM. Acceptability of financial incentives to improve health outcomes in UK and US samples. J Med Ethics 2011;37:682-7.

34 Priebe S, Sinclair J, Burton A, Marouqka S, Larsen J, Firn M, et al. Acceptability of offering financial incentives to achieve medication adherence in patients with severe mental illness: a focus group study. $J$ Med Ethics 2010;36:463-8.

35 World Health Organization. The ICD-10 classification of mental and behavioural disorders: diagnostic criteria for research: WHO, 1993

36 Guy W. National Institute of Mental Health (US). Psychopharmacology Research Branch. Division of Extramural Research Programs. ECDEU assessment manual for psychopharmacology. US Dept. of Health, Education, and Welfare, Public Health Service, Alcohol, Drug Abuse, and Mental Health Administration, National Institute of Mental Health, Psychopharmacology Research Branch, Division of Extramural Research Programs, 1976

37 Priebe S, McCabe R, Bullenkamp J, Hansson L, Lauber C, Martinez-Leal R, et al. Structured patient-clinician communication and 1-year outcome in community mental healthcare - cluster randomised controlled trial. Br J Psychiatry 2007;191:420-6.

38 Priebe S, Golden E, McCabe R, Reininghaus U. Patient-reported outcome data generated in a clinical intervention in community mental health care-psychometric properties. BMC Psychiatry 2012;12:113.

39 Glover GR, Leese M, McCrone P. More severe mental illness is more concentrated in deprived areas. Br J Psychiatry 1999;175:544-8.

40 Stata statistical 10.1 [program]. College Station, TX: StataCorp, 2009

41 Ryan P. Random allocation of treatments in blocks. Stata Tech Bull 1998;41:43-6.

Accepted: 28 August 2013

Cite this as: BMJ 2013;347:f5847 
This is an Open Access article distributed in accordance with the Creative Commons Attribution Non Commercial (CC BY-NC 3.0) license, which permits others to distribute, remix, adapt, build upon this work non-commercially, and license their derivative works on different terms, provided the original work is properly cited and the use is non-commercial. See: http://creativecommons.org/licenses/by-nc/3.0/. 


\section{Tables}

Table 1 | Characteristics of participants at baseline. Values are numbers (percentages) unless stated otherwise

\section{Characteristics}

Mean (SD) age (years)

Men

Mean (SD) years of education

Ethnicity:

White

Black

Asian

Mixed and other

Living situation:

Married or cohabiting

Independent accommodation

Living alone

Paid employment

Receiving benefits

Living in high deprivation area (MINI score)

Diagnosis:

Schizophrenia

Schizoaffective disorder

Bipolar disorde

Other psychosis disorder

Other diagnosis

Clinical history:

Mean (SD) duration of illness (years)

$\geq 1$ hospital admissions in past year

CTO at time of randomisation

Mean (SD) average prescription cycle during baseline

\begin{tabular}{cccc}
$0(0)$ & $43.7(9.8)$ & $44.4(9.6)$ & $42.7(10.2)$ \\
\hline $0(0)$ & $105(74)$ & $59(76)$ & $46(73)$ \\
\hline $29(21)$ & $11.0(1.6)$ & $10.9(1.7)$ & $11.2(1.5)$ \\
\hline $3(2)$ & & & $34(57)$ \\
& $83(60)$ & $49(63)$ & $14(23)$ \\
\hline & $31(22)$ & $17(22)$ & $4(7)$ \\
\hline & $15(7)$ & $5(6)$ & $8(13)$
\end{tabular}

Missing data Total $(n=141)$ Intervention group $(n=78)$ Control group $(n=63)$

MINIl=mental illness needs index; CTO-community treatment order.

$3(2)$

$18(13)$

$8(10)$

$10(16)$

$39(28)$

$102(74)$

$53(68)$

49 (83)

20 (14) $75(62)$

$41(62)$

34 (62)

$3(2) \quad 4(3)$

$6(4)$

4 (3)

$3(4)$

1 (2)

$0(0)$

76 (99)

$58(100)$

$46(73)$

$110(78)$

$4(82)$

$61(78)$

$52(82)$

8 (12)

$17(12)$

$9(12)$

$8(12)$

6 (8)

$1(2)$

$7(5)$

$3(2)$

$1(<1)$

2 (2)

$0(0)$

$1(2)$

$1(2)$

$\mathrm{MINI}=$ mental illness needs index; CTO=community treatment orde 


\begin{tabular}{|c|c|c|c|c|c|c|c|c|}
\hline \multirow[b]{2}{*}{ Outcomes and period } & \multicolumn{2}{|c|}{ Intervention group } & \multicolumn{2}{|c|}{ Control group } & \multirow{2}{*}{$\begin{array}{c}\text { No of } \\
\text { patients in } \\
\text { multivariable } \\
\text { analysis }\end{array}$} & \multirow{2}{*}{$\begin{array}{c}\text { Adjusted } \\
\text { effect } \\
\text { estimate* }^{\star} \\
\text { (intervention } \\
v \text { control) } \\
(95 \% \mathrm{Cl})\end{array}$} & \multirow[b]{2}{*}{$P$ value } & \multirow[b]{2}{*}{ ICC } \\
\hline & $\begin{array}{c}\text { No of } \\
\text { patients }\end{array}$ & Mean (SD) & $\begin{array}{c}\text { No of } \\
\text { patients }\end{array}$ & Mean (SD) & & & & \\
\hline \multicolumn{9}{|l|}{ Primary outcome } \\
\hline \multicolumn{9}{|l|}{$\begin{array}{l}\text { Adherence level to depot } \\
\text { treatment: }\end{array}$} \\
\hline Baseline & 72 & $69 \%(16 \%)$ & 55 & $67 \%(16 \%)$ & 123 & \multirow{2}{*}{$\begin{array}{l}11.5 \%(3.9 \% \\
\text { to } 19.0 \%) \dagger\end{array}$} & \multirow[t]{2}{*}{0.0003} & \multirow[t]{2}{*}{0.28} \\
\hline One year study period & 75 & $85 \%(15 \%)$ & 56 & $71 \%(22 \%)$ & & & & \\
\hline \multicolumn{9}{|l|}{ Secondary outcomes } \\
\hline \multicolumn{9}{|l|}{$\begin{array}{l}\text { Subjective quality of life } \\
\text { (DIALOG mean score): }\end{array}$} \\
\hline Baseline & 51 & $4.4(0.9)$ & 34 & $4.9(1.0)$ & 57 & \multirow{2}{*}{$\begin{array}{c}0.71(0.26 \text { to } \\
1.15) \ddagger\end{array}$} & \multirow[t]{2}{*}{0.002} & \multirow[t]{2}{*}{$<0.001$} \\
\hline End of study period & 56 & $5.2(0.8)$ & 32 & $4.9(1.0)$ & & & & \\
\hline \multicolumn{9}{|l|}{$\begin{array}{l}\text { Satisfaction with medication } \\
\text { (one item mean score): }\end{array}$} \\
\hline Baseline & 51 & $4.9(1.5)$ & 34 & $5.0(1.5)$ & 57 & \multirow{2}{*}{$\begin{array}{c}0.26(-0.74 \text { to } \\
1.27) \dagger\end{array}$} & \multirow[t]{2}{*}{0.610} & \multirow[t]{2}{*}{0.51} \\
\hline End of study period & 56 & $5.6(1.5)$ & 32 & $5.3(1.3)$ & & & & \\
\hline \multicolumn{9}{|l|}{$\begin{array}{l}\text { Outcomes in intervention } \\
\text { group: }\end{array}$} \\
\hline $\begin{array}{l}\text { No of voluntary hospital } \\
\text { admissions }\end{array}$ & 78 & $0.15(0.49)$ & 60 & $0.13(0.43)$ & & & & \\
\hline $\begin{array}{l}\text { No of involuntary hospital } \\
\text { admissions }\end{array}$ & 78 & $0.31(0.96)$ & 60 & $0.27(0.63)$ & & & & \\
\hline \multicolumn{9}{|l|}{ Adverse events } \\
\hline No of suicide attempts & 77 & $0.13(0.41)$ & 58 & $0.10(0.41)$ & & & & \\
\hline No of violent incidents & 77 & $0.25(0.78)$ & 58 & & & & & \\
\hline No of police arrests & 77 & $0.18(0.53)$ & 57 & & & & & \\
\hline \multicolumn{9}{|l|}{$\begin{array}{l}\text { No of patients achieving at } \\
\text { least } 95 \% \text { adherence: }\end{array}$} \\
\hline Baseline & 72 & $5(7 \%)$ & 55 & $1(2 \%)$ & 123 & \multirow{2}{*}{$\begin{array}{c}8.21(2.00 \text { to } \\
33.67) \S\end{array}$} & \multirow[t]{2}{*}{0.003} & \multirow[t]{2}{*}{0.04} \\
\hline One year study period & 75 & $21(28 \%)$ & 56 & $3(5 \%)$ & & & & \\
\hline \multicolumn{9}{|c|}{$\begin{array}{l}\text { No of patients achieving } \\
\text { clinical improvement on CGI: }\end{array}$} \\
\hline End of study period & 57 & $33(58 \%)$ & 44 & $18(41 \%)$ & 96 & $\begin{array}{c}2.73(0.64 \text { to } \\
11.59) \S\end{array}$ & 0.174 & 0.44 \\
\hline
\end{tabular}

CGI=clinical global improvement; ICC=model based intracluster correlation coefficient.

*Each model adjusted for baseline measure of outcome (except when clinical global improvement was outcome), mental illness needs index score category (low $v$ high), and average treatment cycle during baseline, and includes a random effect for team.

†Adjusted difference in means ( $95 \% \mathrm{Cl}$ ) from linear mixed effects model.

$\ddagger$ Adjusted difference in means from random effects model fitted by generalised least squares.

§Adjusted odds ratio $(95 \% \mathrm{Cl})$ from logistic mixed effects model. 


\section{Figure}

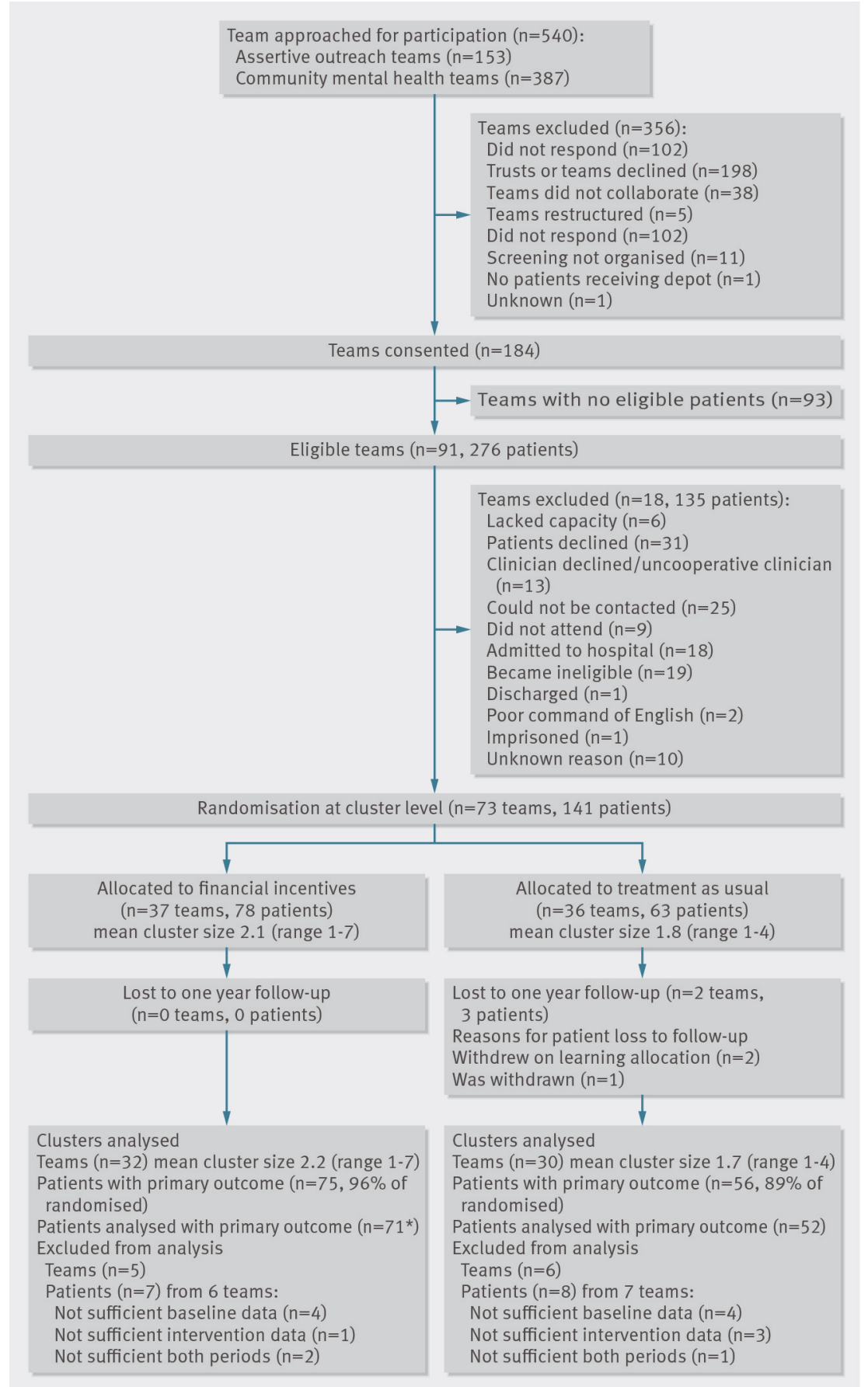

Flow of participants through study 